\title{
Molecular Targeting of Notch Signaling Pathway by DAPT in Human Ovarian Cancer: Possible Anti Metastatic Effects
}

\author{
Maryam Akbarzadeh', Maryam Majidinia², Sedigheh Fekri Aval ${ }^{3}$, Soltanali \\ Mahbub $^{1}$, Nosratollah Zarghami ${ }^{3,4 *}$
}

\begin{abstract}
Background: Ovarian cancer is one of the most important gynecological malignancies, causing significant mortality. Recently, there has been extensive attention to the involvement of signaling cascades in its initiation/progression. In this study, we focused on the possible role of Notch signal transduction in proliferation and metalloproteinase 2 and 9 function in human ovarian cancer OVCAR-3 cells. Methods: MTT proliferation assays were used to evaluate effects of a DAPT inhibitor on cell proliferation. For measurement of Hes-1 mRNA levels, quantitative reverse transcription polymerase chain reaction (qRT-PCR) was applied following $48 \mathrm{~h}$ incubation with the inhibitor. In addition, metalloproteinase (MMPs) activity was assessed by zymography. Results: Inhibition of Notch signaling resulted in a significant reduction in OVCAR-3 cell proliferation. Additionally, DAPT treatment of cells significantly decreased Hes- 1 mRNA levels $(p<0.05)$ as well as activity of MMP-2 and $-9(p<0.05)$. Conclusion: Our results suggested that suppression of Notch signaling by a specific inhibitor can effectively decrease proliferation and the potential for metastasis of OVCAR-3 cells via a reduction in the activity of metalloproteinases 2 and 9. Thus, pharmacological targeting of the Notch signaling pathway could be a promising future treatment for ovarian cancer.
\end{abstract}

Keywords: Notch signaling pathway- metalloproteinases- ovarian cancer

Asian Pac J Cancer Prev, 19 (12), 3473-3477

\section{Introduction}

Ovarian cancer, as one of the most important leading causes of death in gynecological malignancies, is a high heterogeneous and complex neoplasm in histology, biological properties, as well as the cellular origin (Smolle et al., 2013). Epithelial ovarian cancer is the most lethal form in the world and OVCAR-3 cell line is a highly metastatic and drug-resistant human carcinoma cell line, thus it is an appropriate model system to study the effect and mechanisms of various anticancer agents (Beaufort et al., 2014). Because of the non-specific symptoms at the initial, this malignancy is usually diagnosed at advance stages, with invasive and metastatic phenotype (Arend et al., 2013). In spite of a huge effort in elucidating the molecular alternations occurred in this type of malignancy, there is still a challenge in treating ovarian cancer (Block et al., 2015). Hence, insights into key signaling pathways that are aberrantly upregulated in ovarian cancer and play a major function in the tumor progression, recurrence, and metastasis may lead to novel therapies that can improve the treatment strategies for these women (Mabuchi et al.,
2015). The Notch signaling cascade plays pivotal roles in the cell proliferation, differentiation, development, and homeostasis (Shih and Wang, 2007). This signaling pathway is activated upon interaction of Notch receptors (Notch 1-4) to ligands (Delta-like-1, -3, -4, Jagged-1 or -2) (Yabuuchi et al., 2013). The interaction between receptor and ligand results in the proteolytic cleavage of the Notch receptors catalyzed by a member of a disintegrin and metalloproteinase family (ADAM-10 or - 17) of proteases and $\gamma$-secretase, and release of the intracellular domain of the Notch (NICD). The Notch ICD translocates to the nucleus, binds to the transcriptional repressor protein $\mathrm{C}$ binding factor 1 (CBF1; also known as RBP-Јк) or CSL and converts CBF1 from a transcriptional repressor to an activator and formes CBF1-NICD-MAML1 complex thus functions as a transcriptional activator, promotes expression of a number of downstream target genes such as Hes and Hey family (Leong and Karsan, 2006; Aval et al., 2017). These target genes are the best-characterized targets of this transcriptional activation complex and suppress the transcription of downstream targets such as cyclindependent kinase inhibitor p27Kip1, involved in a broad

${ }^{1}$ Department of Biochemistry, Higher Education Institute of Rab-Rashid, ${ }^{3}$ Department of Medical Biotechnology, Faculty of Advanced Medical Sciences, ${ }^{4}$ Department of Clinical Biochemistry and Laboratory Medicine, Faculty of Medicine, Tabriz University of Medical Sciences, Tabriz, ${ }^{2}$ Solid Tumor Research Center, Urmia University of Medical Science, Urmia, Iran. *For Correspondence: Zarghami@tbzmed.ac.ir 
variety of molecular and developmental mechanisms such as those with direct function in the control of cell identity, proliferation and apoptosis (Kageyama et al., 2015; Murata et al., 2005). They function as DNAbinding transcriptional repressors that control cell fate decisions in several contexts (Kageyama et al., 2015). Hence, dysregulation of Notch signaling pathways is an important pathogenic event in various malignancies, including ovarian cancer (Takebe et al., 2014). As reported in The Cancer Genome Atlas (TCGA) Project, which analyzes the most common molecular abnormalities in ovarian cancer, Notch signaling alteration occurred in $22 \%$ of analyzed tumors (Network, 2011). On the other hand, the Notch signaling pathway modulates transcription factors such as NF- $\kappa$ B family members and its targets matrix metalloproteinases (MMP)-2 and 9. Thereby, the Notch signaling pathway is involved in the tumor invasion and metastasis processes by the regulation of MMP-2 and MMP-9 (Liang et al., 2014; Groeneweg et al., 2014). In this study, we first examined the constitutive activity of Notch signaling pathways in OVCAR-3 cell lines followed by inhibition by the specific inhibitor, DAPT, in order to define the functional relevance of these pathways in OVCAR-3 cells. Finally, we assessed the effects of suppression of Notch signaling pathway on the metalloproteinase activity in OVCAR-3 cells.

\section{Materials and Methods}

\section{Cell culture}

Human ovarian cancer OVCAR-3 cells were obtained from Pasteur Institute Cell Culture Collection (Tehran, Iran), and was grown in RPMI 1640 (Gibco; Invitrogen, USA) supplemented with $10 \%$ FBS (Gibco; Invitrogen, USA) and 100 units/ml penicillin/streptomycin (Biowest Nuaillè, France) and maintained in a humidified incubator at $37^{\circ} \mathrm{C}$ in $5 \% \mathrm{CO} 2$. The cells were passaged every $2-3$ days to maintain exponential growth. To determine the effects of inhibition of the Notch signaling pathway with a specific inhibitor, DAPT (N-[N-(3,5-difluorophenacetyl)Lalanyl]- S-phenylglycine t-butyl ester) (Cayman Chemical; Ann Arbor, MI, USA), on OVCAR-3 cells proliferation, 10,000 cells/well were seeded in 96-well plates. Then, increasing concentrations of DAPT (up to $250 \mathrm{nM}$ ) were applied. Cells were incubated with media containing these agents for $48 \mathrm{~h}$.

\section{Cell proliferation assay}

Cell proliferation was assessed by the MTT assay. The MTT assay, as a colorimetric cell proliferation assay, is based on the reduction of a soluble yellow tetrazolium salt [3-(4,5-dimethylthiazol-2-yl) 2,5-diphenyl tetrazolium bromide] (MTT) to a blue formazan crystal by mitochondrial succinate dehydrogenase activity of viable cells. Experiments for each group were performed in triplicate and with a blank control. After treatment with different concentration of inhibitor, the medium was removed and $200 \mu \mathrm{l}$ RPMI-1640 medium supplemented with $10 \%$ FBS and $10 \%$ MTT $(5 \mathrm{mg} / \mathrm{ml})$ was added. After incubation for another $4 \mathrm{~h}$, the supernatant was carefully removed from the wells, the formazan product was dissolved in $1 \mathrm{~mL}$ dimethylsulphoxide (DMSO), and absorbance was measured at $570 \mathrm{~nm}$ with a micro plate reader (Biotek, ELx 800, USA). Plots of cytotoxicity index $(\% \mathrm{CI}=(1-((\mathrm{OD}$ treated $) /(\mathrm{OD}$ control $)) \times 100)$ versus different concentrations of an inhibitor of the signaling pathway were drawn. $\mathrm{IC}_{50}$ was determined from each plot by calculating the slop and intercept. As well, for proliferation assay process, $24 \mathrm{~h}$ after seeding (day 0 ) in 6-well plates cells were treated with $0.1 \mu \mathrm{M}$ of DAPT in DMSO as treated group and DMSO without DAPT as vehicle group. Cells of both groups were harvested at different time points and living cells were counted with Trypan exclusion method.

\section{RNA isolation and qRT-PCR analysis}

$48 \mathrm{~h}$ after incubation with different concentrations of DAPT, total RNA was extracted using the EZ-10 Total RNA Minipreps Kit (Bio Basic Inc., Canada). Total extracted RNA was quantified by optical density measurement (A260/A280 ratio) with NanoDrop 1000 Spectrophotometer (Wilmington, DE, USA) and qualified by agarose gel electrophoresis. Total RNA was subjected to the RT reaction using AccuPowerTM cycle script RT premix kit Real-time PCR (Bioneer; Daejeon, Korea) was carried out using the Maxima Syber Green/ ROX qPCR Master Mix (ThermoFisher Scientific; Bremen, Germany) and analyzed on Rotor-GeneTM 6000 system (Corbett Life Science, Mortlake, NSW, Australia). The internal control was the constitutively expressed housekeeping human $\beta$-actin. Primers were designed by published Gene Bank sequences utilizing Beacon DesignerTM 5.01 software and obtained from Pishgam Biotech (Tehran, Iran). Primers for $\beta$-actin were as follows: F: 5' - TCGTGCGTGACATTAAGGAG -3' and R: 5' - AGGAAGGAAGGCTGGAAGAG -3 '. The primers for human Hes-1 were as follows: $F$ 5'- CTTTCTGTCTTGAAACCCCG -3' and R: 5'TCATTTCCAGAATGTCCGC -3'. All experiments were carried out in triplicate, and for each sample assayed, the threshold cycle $(\mathrm{Ct})$ value for the target gene or $\beta$-actin gene was determined. The relative mRNA expression levels of each gene were normalized to the $\beta$-actin expression level, which allowed target cDNA calculation as $2^{-(\Delta \Delta C t)}$

\section{Zymography}

Conditioned media of cells with or without treatment with DAPT were removed and centrifuged. Bradford method (Bio-Rad) was used for the determining protein concentration. For the evaluating of the functional activity of MMP-2 and MMP-9, $30 \mu \mathrm{l}$ supernatants or reaction mixture were mixed with $10 \mu$ l Laemmli sample buffer without any reducing agent. After SDS-PAGE on $10 \%$ polyacrylamide gels containing $0.1 \%$ gelatin, incubation with $2.5 \%$ Triton $\mathrm{X}-100$ for $30 \mathrm{~min}$ resulted in the removing of SDS from the gel. Then the gels were soaked in incubation buffer $(50 \mathrm{mM}$ Tris- $\mathrm{HCl}, 150 \mathrm{mM} \mathrm{NaCl}$, $10 \mathrm{mM} \mathrm{CaCl}_{2}$ and $0.5 \mathrm{mM} \mathrm{ZnCl}_{2}$, and $\mathrm{pH} 7.5$ ) for $24 \mathrm{~h}$ at $37^{\circ} \mathrm{C}$, and stained with Coomassie blue R $250(0.1 \%)$. Proteolytic activities of latent and activated gelatinase were visualized as clear bands against the blue background 
of stained gelatin.

\section{Statistical analysis}

The statistical calculations were performed with SPSS 16.0 software (SPSS, Chicago, IL, USA). Student's t-test was used for a comparison.

\section{Results}

The proliferation of OVCAR-3 cells in presence of DAPT was examined through MTT assay and confirmed with cell counting using the Trypan blue exclusion method. As shown in Figure 1, compared to the blank control, DAPT significantly reduced cells proliferation in a dose-dependent manner after $48 \mathrm{~h}(\mathrm{p}<0.05)$. The $\mathrm{IC}_{50}$ values for DAPT were $160 \pm 1 \mathrm{nM}$. Five days of treatment with DAPT decreased proliferation of OVCAR-3 cells compared to vehicle (Figure 2). Analysis of the two groups together showed a significant decrease of cell number in the DAPT treated samples at days 3,4 , and $5(\mathrm{p}<0.05)$. To approve that the effects of inhibitor are mediated by the inhibition of Notch signaling pathway, we examined the effect of DAPT on Hes-1 mRNA expression in OVCAR-3 cells by qRT-PCR, as a key gene of this signaling pathway. A single peak in the melting curve confirmed the purity and specificity of the amplified PCR fragments (Figure 3a and 3b). As shown in Figure 3c, compared to untreated cells, DAPT caused a noticeable decrease in Hes-1 expression at mRNA levels $(\mathrm{p}<0.05)$. We used Hes-1 expression levels as an index for validating the efficiency of chemical inhibitor, DAPT. Therefore, Hes-1 has no functional role in our study. Additionally, in order to evaluate the effects of Notch signaling inhibition on the MMP-2 and MMP-9 activity, we used the zymography method. As shown in Figure 4, our zymographic results showed that DAPT causes a significant decrease in the activity of MMP-2 and MMP-9 $(\mathrm{p}<0.05)$.

\section{Discussion}

Accumulating previous studies in the evaluating and identifying the causes and mechanisms involved in the pathogenesis of ovarian cancer to design effective therapies and modern methods for early detection of disease, suggest that variations in genes and important signaling pathways have a function in the pathogenesis of ovarian cancer, as a complex genetic cancer (Kurman and Shih, 2011). In ovarian cancer, the role of the Notch signaling pathway was discovered for the first time in two studies identifying the potential diagnostic markers for

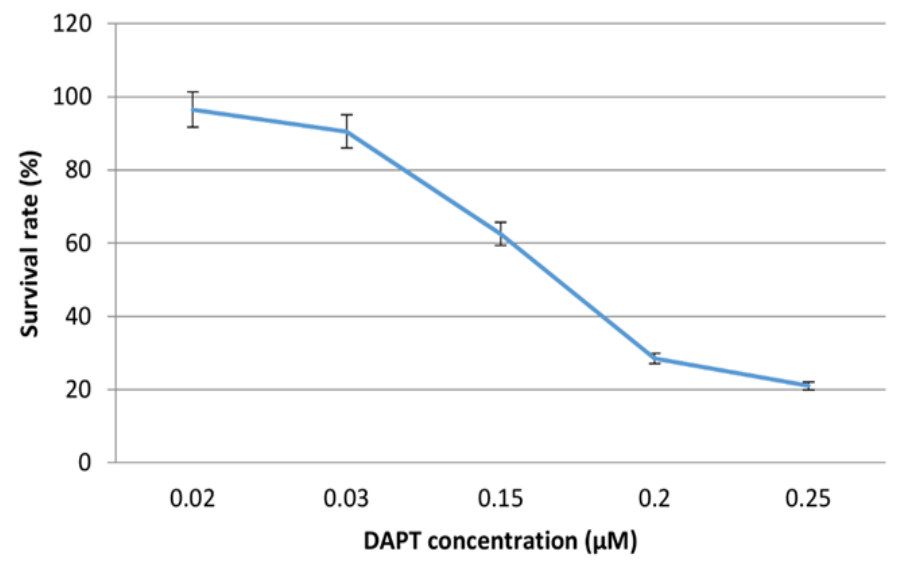

Figure 1. Cytotoxic Effects of DAPT $(0.02,0.03,0.015,0.2,0.25 \mu \mathrm{M})$ after $48 \mathrm{~h}$ Incubation in OVCAR-3 Cells. Results are shown as mean $\pm \mathrm{SD}$ for at least 3 independent experiments. $\mathrm{IC}_{50}$ was determined from each plot by calculating the slop and intercept.

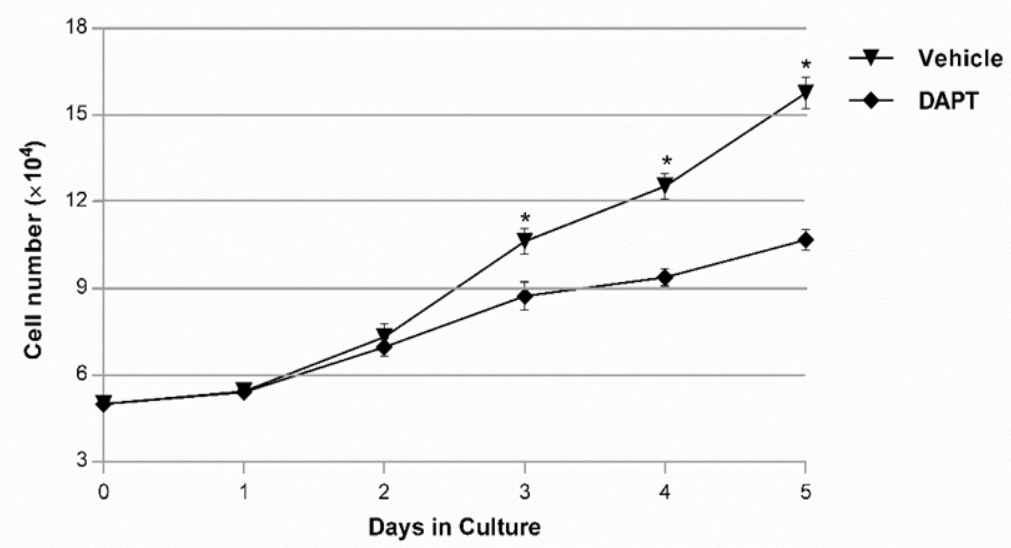

Figure 2. The Cell Proliferation Assay was Assessed on OVCAR-3 Cells Treated with the DAPT or Vehicle and then Living Cells were Counted with Trypan Exclusion Method. A comparison of two groups showed a significant difference after 3 days. $(* p<0.05)$. 
$\mathbf{a}$

b
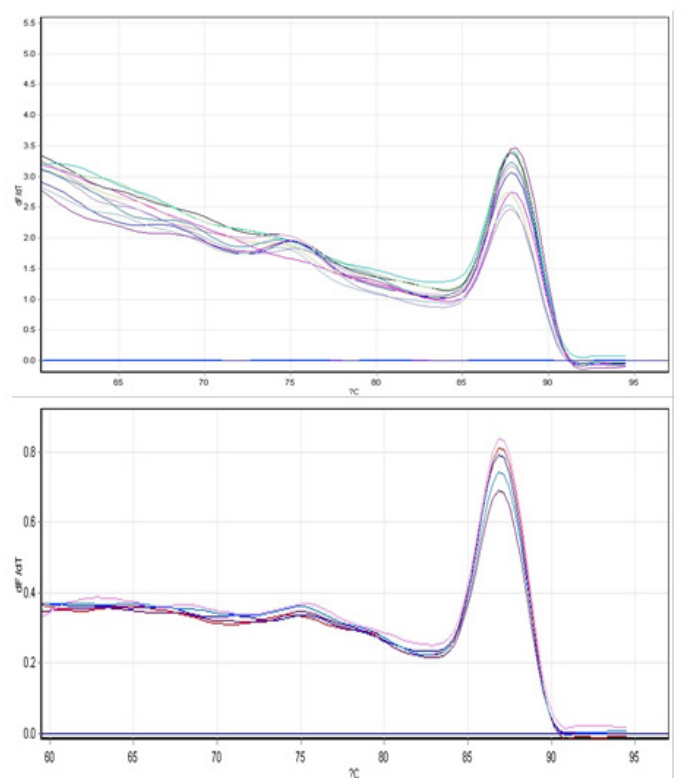

c

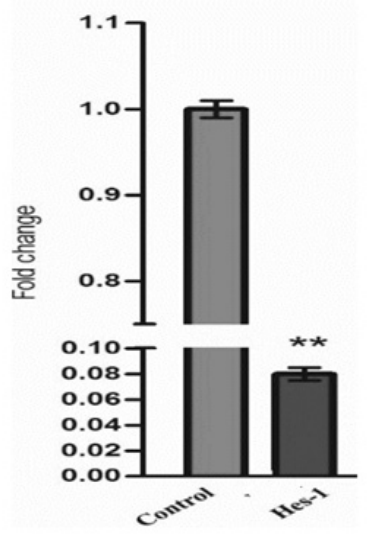

Figure 3. Relative mRNA Expression was Measured by qRT-PCR Using $2^{(-\Delta \Delta C t)}$ Method. (a and b) The melting curve of Hes- 1 and beta actin primers during qRT-PCR process. (c) Determination of mRNA levels Hes-1 in OVCAR-3 Cells upon treatment with different concentration DAPT. The cells were treated with different concentration of DAPT. The results are expressed as mean $\pm \mathrm{SD}$ values from at least 3 independent experiments $(* * p<0.01)$.

a

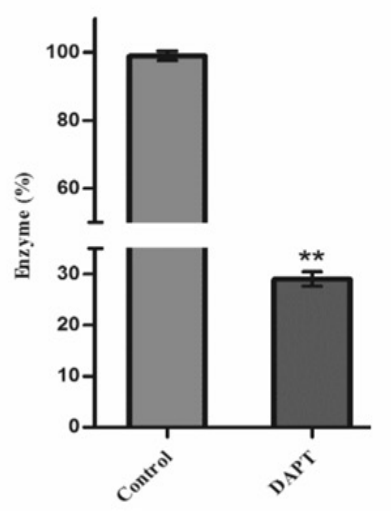

b

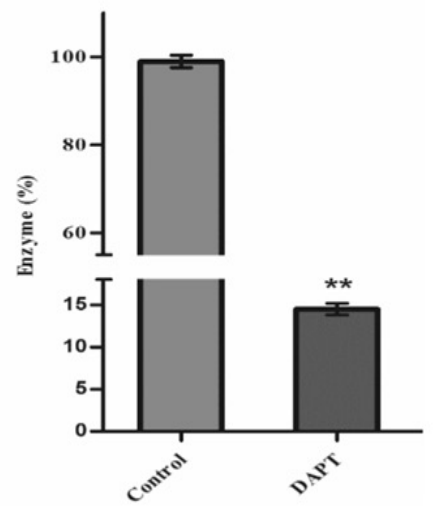

c

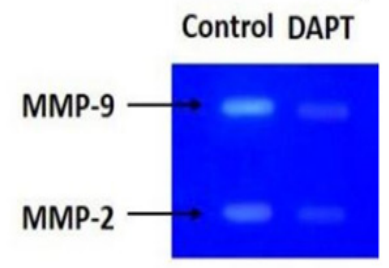

Figure 4. Determination of (a) MMP-2 and (b) MMP-9 Activity Upon the Treatment of OVCAR-3 Cells with DAPT. The results are expressed as mean $\pm \mathrm{SD}$ values from at least three independent experiments $(* * p<0.01)$. (c) DAPT cause a significant decrease in the activity of MMP-2 and MMP-9 $(* p<0.05)$.

ovarian cancer. In one of these studies, overexpression of Notch3 was reported in all cases of analyzed ovarian cancer, while the in the second study, overexpression of Jag2 was reported in cell lines of ovarian cancer in comparison with the control group (Lu et al., 2004; Euer et al., 2005). After that, Park et al., (2006) analyzed the DNA copy number alterations in serous high-grade ovarian cancer by using single nucleotide polymorphism (SNP) array and identified the amplicon of Notch3 loci in 20\% of cases. Notch 3 gene amplification was associated with increased expression of the Notch3 protein (Park et al., 2006). This finding is established by other researchers, who reported the Notch 3 gene amplification in $21 \%$ of ovarian cancers (Nakayama et al., 2007). In addition, recent large-scale genomic and epigenomic analysis of serous high-grade ovarian cancer reported the alternations of the Notch pathway in $22 \%$ of cases with changes in Notch3 (Network, 2011). In another study, it was shown that Jag1 ligand is overexpressed in both ovarian cancer cells and peritoneal mesothelial cells, in that interaction of Jag 1 and Notch 3 resulted in the activation of signaling cascade and stimulates cell proliferation and adhesion. High expression levels of Notch 3 mRNA is associated with bad overall survival and resistance to treatment, and Notch3 protein expression levels significantly are correlated with advanced stage prognosis parameters, lymph node metastasis and distant metastasis (Choi et al., 2008). In a study by Chiaramonte et al., (2015) it was reported that inhibition of the Notch signaling pathway by DAPT decreased the number of ovarian cancer cells. They showed that this effect is due to increased apoptosis and long-term arrest of tumor cells in the G0 / G1 phase. They confirmed that the Notch pathway stimulates the survival and growth of ovarian cancer cells. Similar results were demonstrated by Groeneweg et al., (2014) They showed that ovarian cancer cells treatment with DAPT decreased 
cell proliferation is dose- and time-dependent manner. In agreement with these studies, we showed that inhibition of the Notch signaling pathway with DAPT in OVCAR-3 cells resulted in a significant reduction in cell proliferation in a dose-dependent manner. The effectiveness of therapy was confirmed by measuring changes in gene expression of Hes-1, as a target of the Notch signaling pathway. Therefore, the results showed that the DAPT as a specific inhibitor of Notch signaling pathway, could result in the efficient and specific inhibition of the signaling pathway and by reducing the expression of the key genes of the pathway can cause impaired growth and proliferation of OVCAR-3 ovarian cancer cell line.

The contribution of the Notch signaling pathway in carcinogenesis, progression, invasion and the formation of neurovascular in many malignant tumors was demonstrated by many studies. Notch signaling pathway plays an important role in the regulation of MMP-9 and MMP-2 in tumor invasion and metastasis processes. In a study by Zhou et al., (2012) it was shown that the invasion of hepatocellular carcinoma cells treated with DAPT was decreased. The increase in the DAPT dose as a response to the inhibition of the Notch signaling pathway led to the suppression of MMP-2, MMP-9. These results suggest that the inhibitory effects of DAPT on hepatocellular carcinoma cell line can be partly attributed to the inhibition of MMP-2 and MMP-9. According to these results, Wang et al., (2006) also reported that inhibition of Notch signaling pathway using siRNA decreased the cell invasion, while increased expression of Notch by cDNA transfection increased tumor cell invasion. In addition, the inhibition of Notch signaling pathway has also been shown to inhibit not only the protein and mRNA expression of MMP-9 but suppressed the converting of its inactive form to active form. In accordance with the findings of the mentioned studies, in our study inhibition of the Notch signaling pathway using DAPT resulted in a significant decrease in the activity of MMP-2 and MMP-9 (Wang et al., 2006).

In conclusion, our findings suggest that inhibition of the Notch signaling pathway can effectively reduce OVCAR-3 cells proliferation and metastases via a decrease in the activity of metalloproteinases 2 and 9. Therefore, pharmacological targeting of the Notch signaling pathway could be a promising future treatment of ovarian cancer.

\section{References}

Arend RC, Londoño-Joshi AI, Straughn JM, Buchsbaum DJ (2013). The Wnt//-catenin pathway in ovarian cancer: a review. Gynecol Oncol, 131, 772-9.

Aval SF, Lotfi H, Sheervalilou R, Zarghami N (2017). Tuning of major signaling networks (TGF-beta, Wnt, Notch and Hedgehog) by miRNAs in human stem cells commitment to different lineages: Possible clinical application. Biomed Pharmacother, 91, 849-60.

Beaufort CM, Helmijr JCA, Piskorz AM, et al (2014). Ovarian cancer cell line panel (OCCP): Clinical importance of in vitro morphological subtypes. PLoS One, 9, e103988.

Block MS, Maurer MJ, Goergen K, et al (2015). Plasma immune analytes in patients with epithelial ovarian cancer. Cytokine,
73, 108-13.

Chiaramonte R, Colombo M, Bulfamante G, et al (2015). Notch pathway promotes ovarian cancer growth and migration via CXCR4/SDF1 $\alpha$ chemokine system. Int J Biochem Cell Biol, 66, 134-40.

Choi J-H, Park JT, Davidson B, et al (2008). Jagged-1 and Notch3 juxtacrine loop regulates ovarian tumor growth and adhesion. Cancer Res, 68, 5716-23.

Euer NI, Kaul S, Deissler H, et al (2005). Identification of LICAM, Jagged2 and Neuromedin U as ovarian cancer-associated antigens. Oncol Rep, 13, 375-88.

Groeneweg JW, Foster R, Growdon WB, Verheijen RH, Rueda BR (2014). Notch signaling in serous ovarian cancer. J Ovarian Res, 7, 95.

Kageyama R, Shimojo H, Imayoshi I (2015). Dynamic expression and roles of Hes factors in neural development. Cell Tissue Res, 359, 125-33.

Kurman RJ, Shih I-M (2011). Molecular pathogenesis and extraovarian origin of epithelial ovarian cancer-shifting the paradigm. Hum Pathol, 42, 918-31.

Leong KG, Karsan A (2006). Recent insights into the role of Notch signaling in tumorigenesis. Blood, 107, 2223-33.

Liang W, Gao B, Xu G, et al (2014). Possible contribution of aminopeptidase N (APN/CD13) to migration and invasion of human osteosarcoma cell lines. Int J Oncol, 45, 2475-85.

Lu KH, Patterson AP, Wang L, et al (2004). Selection of potential markers for epithelial ovarian cancer with gene expression arrays and recursive descent partition analysis. Clin Cancer Res, 10, 3291-3300.

Mabuchi S, Kuroda H, Takahashi R, Sasano T (2015). The PI3K/ AKT/mTOR pathway as a therapeutic target in ovarian cancer. Gynecol Oncol, 137, 173-9.

Murata K, Hattori M, Hirai N, et al (2005). Hes1 directly controls cell proliferation through the transcriptional repression of p27Kip1. Mol Cell Biol, 25, 4262-71.

Nakayama K, Nakayama N, Jinawath N, et al (2007). Amplicon profiles in ovarian serous carcinomas. Int $J$ Cancer, $\mathbf{1 2 0}$, 2613-7.

Network CGAR (2011). Integrated genomic analyses of ovarian carcinoma. Nature, 474, 609-15.

Park JT, Li M, Nakayama K, et al (2006). Notch3 gene amplification in ovarian cancer. Cancer Res, 66, 6312-8.

Shih I-M, Wang T-L (2007). Notch signaling, $\gamma$-secretase inhibitors, and cancer therapy. Cancer Res, 67, 1879-82.

Smolle E, Taucher V, Pichler M,et al (2013). Targeting signaling pathways in epithelial ovarian cancer. Int J Mol Sci, 14, 9536-55.

Takebe N, Nguyen D, Yang SX (2014). Targeting notch signaling pathway in cancer: clinical development advances and challenges. Pharmacol Ther, 141, 140-9.

Wang Z, Banerjee S, Li Y, et al (2006). Down-regulation of Notch-1 inhibits invasion by inactivation of nuclear factor- $\kappa \mathrm{B}$, vascular endothelial growth factor, and matrix metalloproteinase-9 in pancreatic cancer cells. Cancer Res, 66, 2778-84.

Yabuuchi S, Pai SG, Campbell NR, et al (2013). Notch signaling pathway targeted therapy suppresses tumor progression and metastatic spread in pancreatic cancer. Cancer Lett, 335, 41-51.

Zhou L, Wang D-S, Li Q-J, et al (2012). Downregulation of the Notch signaling pathway inhibits hepatocellular carcinoma cell invasion by inactivation of matrix metalloproteinase-2 and-9 and vascular endothelial growth factor. Oncol Rep, 28, 874-82.

This work is licensed under a Creative Commons AttributionNon Commercial 4.0 International License.

Aslan Pacific Journal of Cancer Prevention, vol ly $\mathbf{5 4 / /}$ 